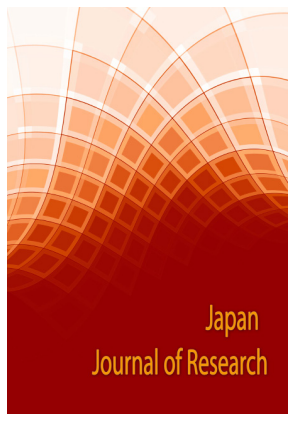

Correspondence

Les Kalman,

Assistant Professor, Restorative Dentistry, Schulich School of Medicine \& Dentistry, Western University, 1151 Richmond Street, London, Ontario, Canada, N6A 3K7 E-mail: ljkalman@icloud.com

- Received Date: 15 Sep 2021

- Accepted Date: 22 Sep 2021

- Publication Date: 05 Oct 2021

Copyright

(c) 2021 Science Excel. This is an openaccess article distributed under the terms of the Creative Commons Attribution 4.0 International license.

\title{
Preliminary assessment of dry electropolishing technology on a novel additive manufactured Ti- 6Al-4V implant abutment
}

\author{
Les Kalman ${ }^{1+*}$ and Ben Kalman ${ }^{2 \dagger}$ \\ ${ }^{1}$ Assistant Professor, Restorative Dentistry, Schulich School of Medicine \& Dentistry, Western University, Ontario, Canada \\ 2BMSc Candidate, Medical Sciences, Western University, Ontario, Canada \\ ${ }^{t}$ These authors contributed equally to this work.
}

\begin{abstract}
Unprocessed components fabricated by metal additive manufacturing (AM) tend to be too rough for immediate implementation and require post-processing to allow for proper physical contact, fit and suitability. Utilization of metal AM for the fabrication of dental prostheses requires a smooth finish to prevent the formation of a biofilm and possible inflammatory responses. Standard polishing protocols utilize hand tools and rotary burs to create a mirror polished finish but may not be possible for some small and intricate devices. Dry electropolishing technology (DET) is an automated process for metal AM parts that employs electropolishing by ion transport using free solid bodies, based in a solid media. This preliminary investigation explored dry electropolishing technology on an additive manufactured Ti-6Al-4V implant abutment. The abutments were fabricated with additive manufacturing in dental grade titanium Ti-64 (titanium 6-aluminum 4-vanadium) using Selective Laser Melting (SLM). Components were photographed with a USB microscope at a magnification of $200 \mathrm{x}$, prior to and after DET. The novel dental abutment was evaluated with magnification, a preclinical assessment and evaluation with radiology. DET provided significant polishing of the pin projections, abutment surface and circumference. There was no tactile evidence of thread slippage or excessive play and there were no microgaps detected. The fit seemed clinically acceptable. DET technology seems to provide a simple and predictable post-processing technique that provides surface polishing with Ti-64 dental implant components that cannot be finished by conventional methods.
\end{abstract}

\section{Introduction}

Computer Aided Milling (CAM), or simply milling, remains the most common method of metal prostheses fabrication in dentistry $[1,2]$. However, several investigators [3,4] have explored novel workflows utilizing metal additive manufacturing (AM) for the fabrication of dental prosthesis. Kalman [5] has fabricated and tested Ti-64 dental implants components, specifically a novel abutment, in Ti64. The workflow employed a hybrid approach to fabrication, AM for production, and subtractive manufacturing for manually creating the threads with a die. Due to the intricate design and small size (Figure 1), standard polishing utilizing rotary instruments was not feasible and other approaches required further investigation.

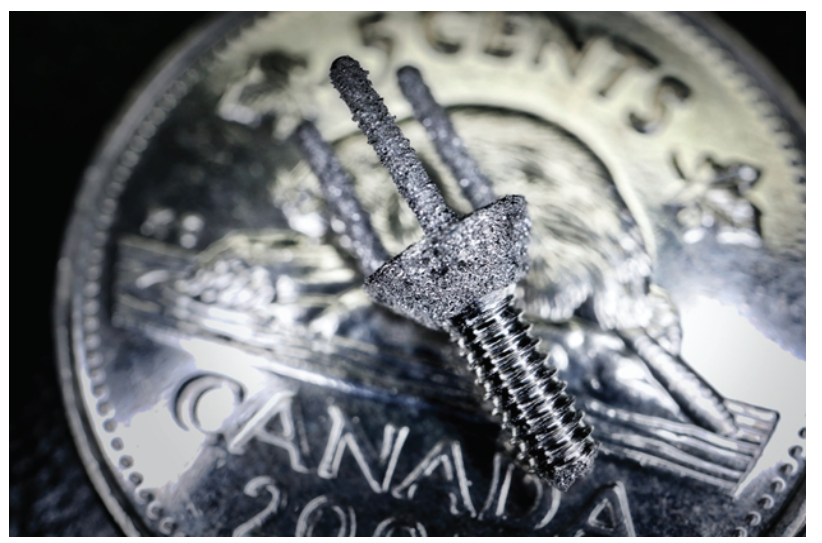

Figure 1. Novel dental implant abutment scaled against a Canadian nickel (with $20 \mathrm{~mm}$ diameter). 
Dental prostheses require a smooth finish to prevent the formation of a biofilm $[6,7,8]$, which can have an unfavourable impact on the oral health of the patient and the outcome of the treatment $[9,10]$. Dental implant components should also exhibit a smooth surface finish, to prevent the accumulation of biofilms $[7,8,11]$ and the development of peri-mucositis and per-implantitis $[12,13]$. Currently, peri-implantitis is evident with $22 \%$ of patients rehabilitated with dental implant therapy [14].

The unprocessed products fabricated by metal AM tend to be too rough for immediate implementation and must be finished to allow proper physical contact, fit and suitability $[15,16]$. As polishing remains a critical step in post-processing of AM devices, especially with medical devices [17,18], there are different approaches to create a smooth surface.

Standard polishing protocols utilize hand tools and rotary burs to remove material and create a mirror polished finish $<$ $1 \mu \mathrm{m} \mathrm{Ra}[19,20]$. The amount of polishing of final components needs to be determined, from brushed or matte to mirror finish. This approach is labour and time intensive, removes material and is limited by the size of the component and geometry. For some devices, this approach is not possible.

Electropolishing is a general method of polishing that involves ion activity to smooth surfaces [21]. Dry electropolishing technology (DET) (DryLyte: GPAInnova, Barcelona, Spain) is a specific automated polishing system for metal parts that employs electropolishing by ion transport using free solid bodies, based in a solid media [22-23]. DET is able to polish different metal surfaces without unwanted scratches or markings and maintains homogeneity across the entire surface of the object [22-23]. It has the ability to provide a smooth finish to complex geometries [22-23]. Although some solution-based polishing methods smooth a surface by removing material from the edges [21], DET takes a different approach as it maintains intentionally sharp edges without causing blunting. The surface of the components become significantly flatter, but the intended shape of the object has not been distorted [22-23].

This preliminary investigation explored an in vitro assessment of dry electropolishing technology on a novel additive manufactured Ti-6Al-4V dental implant abutment.

\section{Materials and methods}

Several dental implant abutment components (Figures 2-4) were fabricated with an additive manufacturing workflow [3] using Ti-64. The components were designed with Fusion 360 software (Autodesk, San Rafael, California, USA), were optimized and printed in dental grade titanium Ti-64 (titanium 6-aluminum 4-vanadium) using Selective Laser Melting (SLM) technology with a Renishaw AM 400 laser melting system (Renishaw PLS, Gloucestershire, United Kingdom). Metallic powder was melted and fused with an average diameter between 30-50 $\mu \mathrm{m}$ [24]. Post-processing involved heat treatment, bead blasting (Ceramic, Zirblast B120, Saint-Gobain ZirPro, France) and fixation with a custom stabilization jig for the thread placement on the final abutment design (Figure 4). The components were not physically polished, due to the small size and intrinsic geometry.

The components were photographed with a USB microscope (Dino-Lite, Torrance, USA) at maximum magnification of 200x, prior to DET. The components were delivered to a DLyte facility and underwent standard DET protocols and were then returned. The polished components were photographed with the same
USB microscope with similar orientations, to evaluate the polish. The finalized version of the novel dental abutment was further evaluated using a preclinical assessment including (1) threading the abutment into an implant analogue and assessing the fit and play (2) by hand torqueing to approximately $15 \mathrm{Ncm}$ and (3) evaluation with a digital radiograph to identify the seating and any microgaps.

\section{Results}

Figure 2A depicts the implant component before DET while Figure $2 \mathrm{~B}$ depicts the component after DET. DET appears to smooth out the surface roughness, i.e. reduce the coarseness, but there is still surface topography.

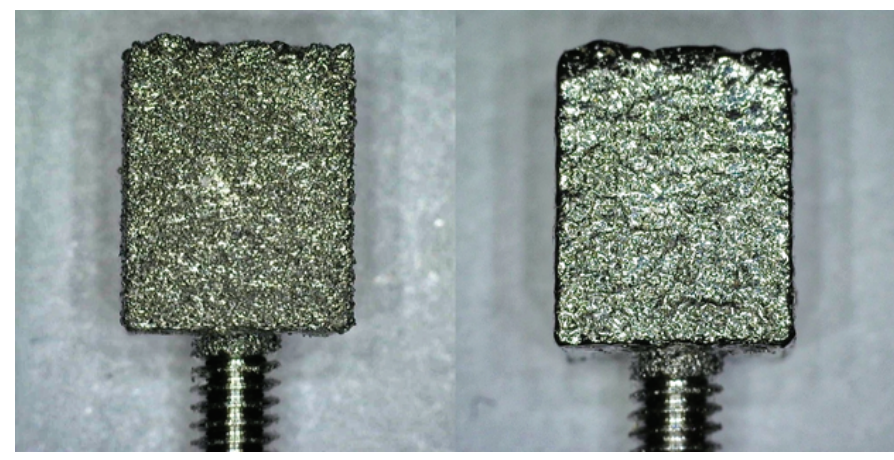

Figure 2. Figures $2 A$ (left) is the implant abutment component before DET and $2 B$ (right) is the component after DET. Width of component is $4.5 \mathrm{~mm}$.

Figure 3A depicts an implant abutment iteration before DET, and after (Figure 3B). DET provided significant polishing of the pin projections and the abutment surface, but minimal polishing of the abutment circumference.

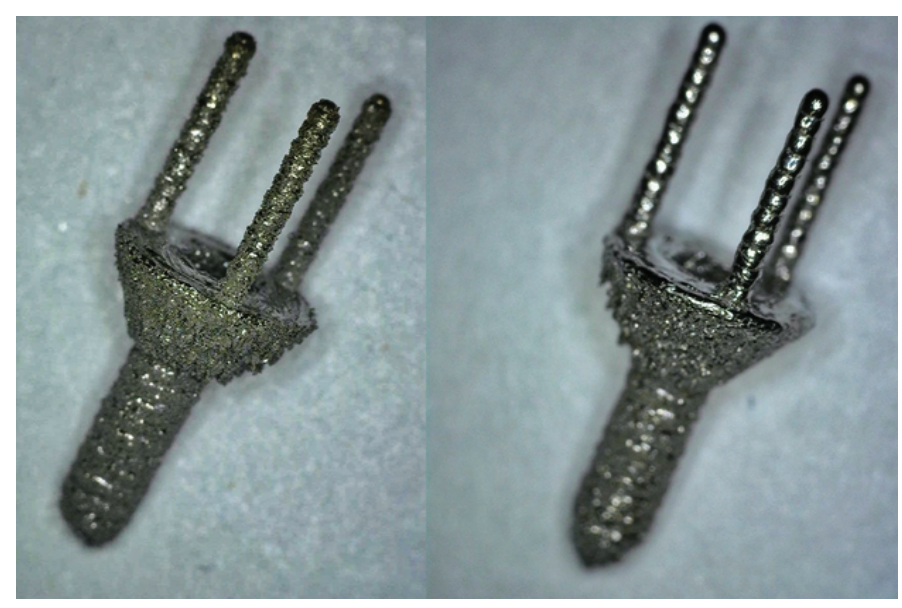

Figure 3. Figures $3 A$ (left) is the implant abutment before DET and $3 B$ (right) is the abutment after DET. Maximum width of abutment is $4.5 \mathrm{~mm}$

Figure 4A depicts the finalized design of implant abutment before DET and Figure $4 \mathrm{~B}$ depicts after the process. DET provided significant polishing of the pin projections, abutment surface and circumference. 


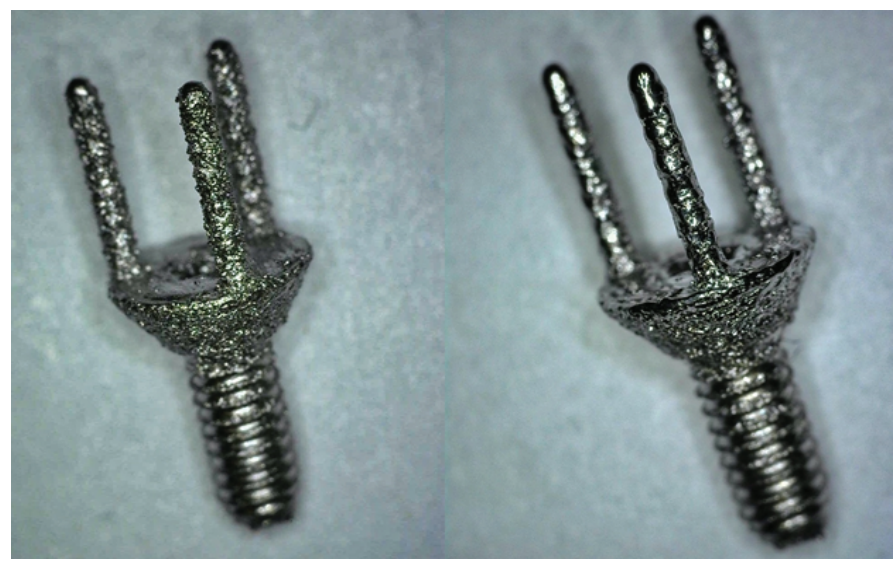

Figure 4. Figures $4 A$ (left) is the finalized implant abutment before DET and $4 B$ (right) is the abutment after DET. Maximum width of abutment is $4.5 \mathrm{~mm}$.

Figure 5 depicts the abutment threads, that torque into a dental implant. The threads seam to remain unaffected by the DET.

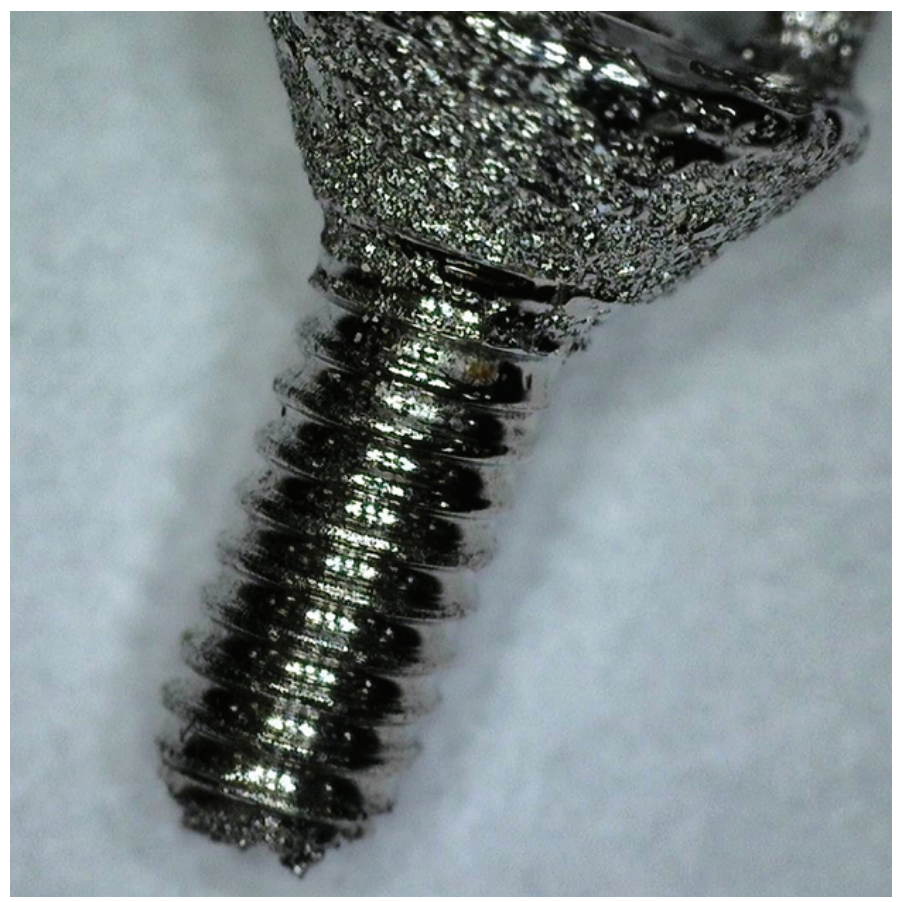

Figure 5. Abutment threads. Width of shaft is $2.1 \mathrm{~mm}$.

Figure 6A depicts the DET polished abutment with an implant analogue. The abutment was hand torqued to approximately 15 $\mathrm{Ncm}$. There was no tactile evidence of thread slippage or excessive play and a dental explorer could not detect any microgaps. The fit seemed clinically acceptable. Figure $6 \mathrm{~B}$ is a radiograph of the DET polished abutment threaded into an abutment. The fit seemed clinically acceptable and unaffected by DET.

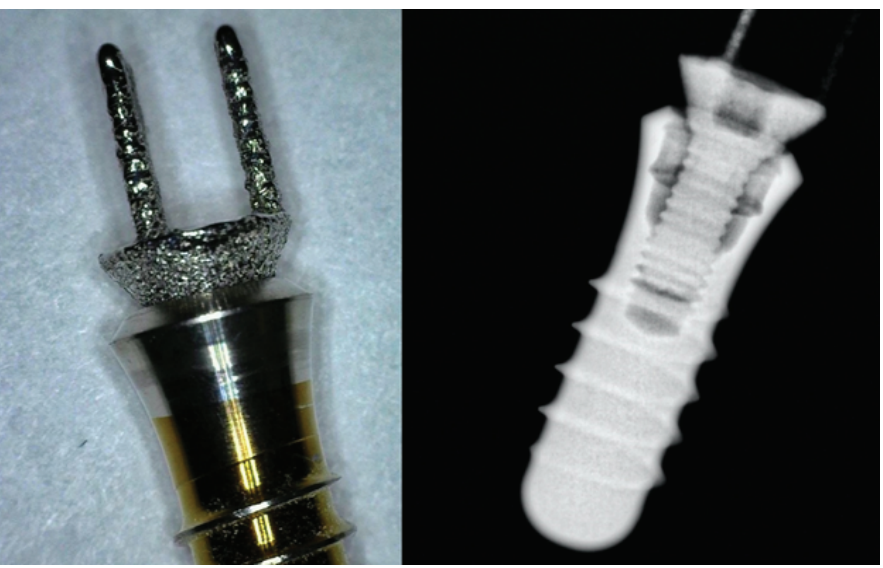

Figure 6. Figures $6 A$ (left) illustrates the post-DET abutment and implant fit and $6 B$ (right) is a radiograph of the post-DET abutment and implant.

\section{Discussion}

DET provides a process to polish AM components that may not be polished through other conventional approaches, as is the case with this investigation. DET seems to provide an adequate surface polish, but still retains the surface topography and does not affect the threading. The threaded component may have been clamped during the DET process. The components would have benefited from an initial abrasive rotary smoothing of the surface to provide a homogeneous finish, which is recommended in the DET protocol, but the component was so small with intrinsic geometry that this was not practical.

As the DET process employs a dental holder, the AM components may require the incorporation of a 'sprue' to permit proper submersion for complete polishing. DET provides polishing for titanium and cobalt-chromium. Although the cost of polishing was waived, the cost of the capital investment of the unit and the automated polishing process should be weighed against the labour costs of time-intensive manual polishing. Time comparison would also have to account for this automated post-processing approach.

An understanding of the specific AM process, which fabricates components with a different micro-structure than milling, is also required to appreciate the pre-process structure of components and how DET may improve the surface polish. If rotary tools and burs are required to establish a homogenous surface, the component design should account for the estimated loss of material. The DET rate of material loss should also be quantified, which may be difficult based on the material and geometry.

Maintaining the surface topography may be desirable in some dental implant components, to maximize retention of prosthesis, while surface finish is required to prevent biofilm accumulation and the development of inflammation.

The surface polish or smoothness would require further research through assessment and evaluation with greater magnification (SEM), a larger sample size, microbiologic and corrosion assessments.

\section{Conclusions}

Dry electropolishing technology seems to provide a surface polish that seems subtle enough to significantly improve the 
finish, without impacting the structure, topography, fit or threading of an abutment with a dental implant.

DET technology seems to provide a simple and predictable post-processing technique that provides surface polishing of a novel additive manufactured Ti-64 dental implant abutment that cannot be finished by conventional methods.

Additive manufacturing is having a significant impact on dentistry and metal AM is beginning to influence implant dentistry. As technological advances continue, metal AM may be considered as an alternative workflow for the fabrication of dental implant components.

\section{Acknowledgements}

We would like to thank the remarkable team at ADEISS for the digital design and additive manufacturing of the implant components and the team at DryLyte for providing the dry electropolishing of the components.

\section{Conflict of interest}

The first author is the inventor of the novel dental implant abutment.

\section{Author contributions}

Conceptualization, LK \& BK.; methodology, LK; investigation, LK \& BK; data curation, LK; writing - original draft preparation, LK \& BK; writing - review and editing, LK \& BK; visualization, LK; supervision, LK. All authors have read and agreed to the published version of the manuscript.

\section{Funding}

This research was funded by the Schulich Dentistry IRG and the International Congress of Oral Implantologists (ICOI) Implant Dentistry Research \& Education Foundation.

\section{References}

1. Yin L, Song XF, Song YL, Huang T, Li J. An overview of in vitro abrasive finishing \& CAD/CAM of bioceramics in restorative dentistry. International Journal of Machine Tools and Manufacture. 2006;46(9):1013-1026.

2. Beuer F, Schweiger J, Edelhoff D. Digital dentistry: An overview of recent developments for CAD/CAM generated restorations. British Dental Journal. 2008;204(9):505-511.

3. Kalman L, Hosein Y, Chimel T. Workflow Development of a 3D Printed Novel Implant Abutment. 3D Printing and Additive Manufacturing. 2019;6(5):235-237.

4. Revilla-León M, Sadeghpour M, Özcan M. A Review of the Applications of Additive Manufacturing Technologies Used to Fabricate Metals in Implant Dentistry. Journal of Prosthodontics. 2020;29(7):579-593.

5. Kalman L. In vitro assessment of a novel additive manufactured titanium implant abutment. J Clin Exp Dent. 2021;13(2):e99-103

6. Rimondini L, Cochis A, Varoni E, Azzimonti B, Carrassi A. Biofilm Formation on Implants and Prosthetic Dental Materials. In: Antoniac I. (eds) Handbook of Bioceramics and Biocomposites. 2015; Springer, Cham.
7. Hannig $\mathrm{M}$, Kriener L, Hoth-Hannig W, Becker-Willinger $\mathrm{C}$, Schmidt H. Influence of nanocomposite surface coating on biofilm formation in situ. Journal of Nanoscience and Nanotechnology. 2007;7(12):4642-4648.

8. Quirynen M, Bollen CML. The influence of surface roughness and surface-free energy on supra- and subgingival plaque formation in man: A review of the literature. Journal of Clinical Periodontology. 1995;22(1):1-14.

9. Gurenlian JR. The Role of Dental Plaque Biofilm in Oral Health. American Dental Hygienists Association. 2007;81(suppl 1),116 LP-116.

10. Yip KHK, Smales RJ. Implications of oral biofilms in medically at risk persons. Journal of Biomedical Research. 2012;26(1):1-7.

11. Xing R, Lyngstadaas SP, Ellingsen JE. Taxt-Lamolle S. \& Haugen HJ. The influence of surface nanoroughness, texture and chemistry of TiZr implant abutment on oral biofilm accumulation. Clinical Oral Implants Research. 2015;26(6):649656.

12. Heitz-Mayfield LJA, Salvi GE. Peri-implant mucositis. Journal of Clinical Periodontology. 2018;45:S237-S245.

13. Dhir S. Biofilm and dental implant: The microbial link. Journal of Indian Society of Periodontology. 2013;17(1):5-11.

14. Derks J, Tomasi C. Peri-implant health and disease. A systematic review of current epidemiology. Journal of Clinical Periodontology. 2015;42(S16):158-S171).

15. Hassanin H, Elshaer A, Benhadj-Djilali R, Modica F, Fassi I. Surface Finish Improvement of Additive Manufactured Metal Parts. Springer, Cham. 2018: 145-164.

16. Gora WS, Tian Y, Cabo AP, et al. Enhancing surface finish of additively manufactured titanium and cobalt chrome elements using laser based finishing. Physics Procedia. 2016;83:258-263.

17. Cunico MWM, Cunico MM, Cavalheiro PM, De Carvalho J. Investigation of additive manufacturing surface smoothing process. Rapid Prototyping Journal. 2017; 23(1):201-208.

18. Kumbhar NN, Mulay AV. Post Processing Methods used to Improve Surface Finish of Products which are Manufactured by Additive Manufacturing Technologies: A Review. Journal of The Institution of Engineers (India): Series C. 2018;99(4):481-487.

19. Ho CMB, Ding H, Chen X, Tsoi JKH, Botelho MG. The effects of dry and wet grinding on the strength of dental zirconia. Ceramics International. 2018;44(9):10451-10462.

20. Happe A, Röling N, Schäfer A, Rothamel D. Effects of different polishing protocols on the surface roughness of Y-TZP surfaces used for custom-made implant abutments: a controlled morphologic SEM and profilometric pilot study. Journal of prosthetic dentistry. 2015;113(5):440-447.

21. Zeidler H, Böttger-Hiller F, Edelmann J, Schubert A. Surface Finish Machining of Medical Parts Using Plasma Electrolytic Polishing. Procedia CIRP. 2016;49:83-87.

22. A revolutionary Dry Electropolishing Technology How it works. (n.d.).

23. Inoxl, S. (n.d.). A New Concept of Polishing DryLyte Technology.

24. Saunders M. Designs for metal AM: a beginner's guide. Renishaw. 2019:1-7. 